Article

\title{
Derivation of Lighthill's Eighth Power Law of an Aeroacoustic Quadrupole in Acoustic Spacetime
}

\author{
Drasko Masovic * (iD and Ennes Sarradj (iD) \\ Technische Universität Berlin, Einsteinufer 25, 10587 Berlin, Germany; ennes.sarradj@tu-berlin.de \\ * Correspondence: drasko.masovic@tu-berlin.de
}

Received: 13 July 2020; Accepted: 2 September 2020; Published: 8 September 2020

\begin{abstract}
Acoustic spacetime is a four-dimensional manifold analogue to the relativistic spacetime with the reference speed of light replaced by the speed of sound. It has been established primarily for the indirect studies of relativistic phenomena by means of their better understood acoustic analogues. More recently, it has also been used for the analytical treatment of sound propagation in various uniform and non-uniform flows of the background fluid. In this paper the analogy is extended and utilized to derive Lighthill's eight power law for sound generation of an aeroacoustic quadrupole. Adding to the existing analogue theory, propagating sound waves are described in terms of a weak perturbation of the background acoustic spacetime metric. The obtained result proves that the acoustic analogy can be extended to cover both weak perturbation of the fluid due to the sound waves and certain sound generation mechanisms, at least in incompressible low Mach number flows.
\end{abstract}

Keywords: acoustic spacetime; Lighthill's aeroacoustic analogy; quadrupole radiation

\section{Introduction}

The fundamental work of M. J. Lighthill on aerodynamic sound and the introduction of the first aeroacoustic analogy present a milestone in the development of aeroacoustics and acoustics in general. In his paper [1] Lighthill not only established the basis for most of the later research in aeroacoustics, but also pointed to several phenomena which were not well understood before his work:

- physical mechanism of sound generation by pure instability in a fluid (in contrast to moving solid bodies or boundaries, which involve forces external to the fluid),

- quadrupole sound radiation in free space,

- inefficiency of kinetic-to-acoustic energy conversion in the flow of fluid, and

- $\quad$ power scaling for an aeroacoustic quadrupole source, which Lighthill was able to derive even without an accurate description of the flow, and its relation with the other two main types of sources-monopole and dipole.

Before Lighthill's explanation of aeroacoustic sound generation, quadrupole radiation had only a marginal importance in classical mechanics. However, parallel to the development of aeroacoustics, quadrupole radiation took a central place in the relativistic theory of gravitational waves [2], small curvatures of spacetime which cannot be generated by monopole or dipole sources.

The difference between classical acoustics, defined in separate Newtonian space and time, and the theory of relativity, which is based on the concept of a four-dimensional spacetime, is largely diminished by the introduction of analogue acoustic spacetime. The first observations on the similarity between special relativity and sound propagation date back to W. Gordon [3]. Since then, Lorentz transformations have been successfully used for the problems of sound propagation in uniform mean flows $[4,5]$. The analogy with general relativity and curved spacetime is commonly attributed to W. G. Unruh, who used it primarily for studying Hawking radiation by means of much better 
understood acoustics in transonic flows [6]. The analogy was further developed and promoted by M. Visser and C. Barceló in their studies of what is known as analogue gravity [7] or analogue models of general relativity [8]. The authors have shown that sound propagation in inhomogeneous flows of the background fluid can be described with differential geometry of a curved spacetime when the speed of light is replaced by the speed of sound. Different metric tensors of so defined acoustic spacetime can efficiently capture sound propagation effects such as convection and refraction. In particular, wave operator of the Pierce equation [9] can be obtained [10] and even more general forms of metrics are discussed by Bergliaffa et al. [11].

Despite the analogy between the two analogue background spacetimes and the occurrence of quadrupole radiation in both of them in the absence of external forces, no attempts have been made to express aeroacoustic sound generation in the relativistic framework. In this work, we show that Lighthill's power law for aeroacoustic quadrupole in an inviscid flow can be derived from the acoustic spacetime analogy. Thus we also prove that the analogy can be used not merely for sound propagation, but also for aeroacoustic sound generation. In fact, since Lighthill's analogy assumes quiescent fluid outside the source region, we can consider the simplest flat (Minkowski) background acoustic spacetime, which is weakly perturbed by the waves. The purely geometric perturbation representing propagating sound waves is caused by the fluid motion inside the source region. The linearized relativistic theory, suitable for its description, is presented in the next section. The sound generation mechanism is considered in Section 3 and the eighth power law for the quadrupole source is obtained. Unlike Lighthill's aeroacoustic analogy, which is an exact reformulation of the governing equations of fluid dynamics, the eighth power law holds only for incompressible (low Mach number) flows in the source region and in the absence of a significant acoustic feedback. Indeed, these conditions allow a purely kinematic analogy with general relativity to be established and applied for capturing sound generation in the following analysis. Several relevant outcomes which follow from the derivation of the eighth power law from the analogy are discussed in the concluding Section 4, which also gives suggestions for future studies.

\section{Waves and Motion in Acoustic Spacetime}

Adopting the mixed signature $[-+++]$, the simplest flat Minkowski (acoustic) spacetime is characterized by the second-order metric tensor

$$
\eta^{\alpha \beta}=\left[\begin{array}{cccc}
-1 & 0 & 0 & 0 \\
0 & 1 & 0 & 0 \\
0 & 0 & 1 & 0 \\
0 & 0 & 0 & 1
\end{array}\right] .
$$

Here, and in the rest of the text, Greek letters denote four-dimensional components $(\alpha, \beta, \cdots=$ $0 \ldots 3$, where 0 corresponds to the time coordinate) and Latin letters are used for the spatial components only (for example, $i=1 \ldots 3$ ). Therefore, $x^{0}=c_{0} t$, where $c_{0}$ is the constant reference speed of waves (here the speed of sound in the quiescent fluid at infinity) and $x^{1}$ to $x^{3}$ are spatial coordinates. The associated d'Alembertian is the classical wave operator. For a scalar $\phi$,

$$
\square \phi=\eta^{\alpha \beta} \phi, \alpha \beta=\phi^{, \alpha}, \alpha=\left(-\frac{1}{c_{0}^{2}} \frac{\partial^{2}}{\partial t^{2}}+\nabla^{2}\right) \phi,
$$

since $_{0}=\frac{\partial}{\partial x^{0}}=\frac{1}{c_{0}} \frac{\partial}{\partial t}$ and $\nabla^{2}=\frac{\partial^{2}}{\partial\left(x^{1}\right)^{2}}+\frac{\partial^{2}}{\partial\left(x^{2}\right)^{2}}+\frac{\partial^{2}}{\partial\left(x^{3}\right)^{2}}$. Comma denotes usual derivative with respect to the coordinate which follows it (for example, $\phi, \alpha=\partial \phi / \partial x^{\alpha}$ is gradient of $\phi$ ) and we use Einstein's convention which implies summation over each letter appearing in an expression once as a subscript and once as a superscript [2]. The two positions of the letters correspond to the covariant and contravariant vector bases. Multiplication with $\eta^{\alpha \beta}$ raises the index, as in Equation (2), 
while multiplication with $\eta_{\alpha \beta}$ lowers it. Consequently, four components of vectors $A^{\alpha}$ and $A_{\alpha}=\eta_{\alpha \beta} A^{\beta}$ are equal, apart from the time components which have opposite signs, since $\eta_{\alpha \beta}=\eta^{\alpha \beta}$ has the component $\eta_{00}=-1$.

Next, we suppose that the only disturbance of otherwise flat spacetime outside a spatially confined source region is due to propagating waves. In other words, we assume a quiescent fluid with constant density $\rho_{0}$ and speed of sound $c_{0}$ through which the sound waves propagate. The total metric tensor $g^{\alpha \beta}$ is by definition symmetric and can be written as the sum of $\eta^{\alpha \beta}$ and a weak component $h^{\alpha \beta}$ :

$$
g^{\alpha \beta}=\eta^{\alpha \beta}+h^{\alpha \beta}
$$

with $\left|h^{\alpha \beta}\right| \ll 1$. In the linear approximation it can be shown [2] that there always exists

$$
\bar{h}^{\alpha \beta}=h^{\alpha \beta}-\frac{1}{2} \eta^{\alpha \beta} h^{v}
$$

such that $\left|\bar{h}^{\alpha \beta}\right| \ll 1$ and

$$
\bar{h}^{\alpha \beta}, \beta=0 .
$$

Here, $h^{v}{ }_{v}$ is the trace of $h^{\alpha \beta}$ and Equation (5) is called the Lorenz gauge condition. If the condition is not satisfied directly by $\bar{h}^{\alpha \beta}$ from Equation (4) in a certain frame, one can introduce a small change of coordinates (gauging) $x^{\alpha} \rightarrow x^{\alpha}+\xi^{\alpha}$ which transforms the metric as $h^{\alpha \beta} \rightarrow h^{\alpha \beta}-\xi^{\alpha, \beta}-\xi^{\beta, \alpha}$ such that

$$
\bar{h}^{\alpha \beta}=h^{\alpha \beta}-\frac{1}{2} \eta^{\alpha \beta} h^{v}{ }_{v}-\xi^{\alpha, \beta}-\xi^{\beta, \alpha}+\eta^{\alpha \beta} \xi^{v}{ }^{v}
$$

does satisfy it. For future use, we should also note that $\bar{h}^{\alpha}{ }_{\alpha}=-h^{\alpha}{ }_{\alpha}$ and Equation (4) can be inverted to

$$
h^{\alpha \beta}=\bar{h}^{\alpha \beta}-\frac{1}{2} \eta^{\alpha \beta} \bar{h}_{v}^{v}
$$

Relativistic equations which relate the weak perturbation of spacetime with its source, the stress-energy tensor $T^{\alpha \beta}$, are the linearized Einstein field equations [2,12],

$$
\square \bar{h}^{\alpha \beta}=-\frac{2 k G}{c_{0}^{4}} T^{\alpha \beta}
$$

in which dimensionless $k$ (not to be confused with wave number) and $G\left(\mathrm{in} \mathrm{m}^{3} /\left(\mathrm{kg} \mathrm{s}^{2}\right)\right.$ ) are constants. The instability of $T^{\alpha \beta}$, which is associated with motion of matter (or energy) in the absence of boundaries of spacetime, is the source of the fluctuations $\bar{h}^{\alpha \beta}$, which are considered to be too weak to affect the source mechanism. Such decoupling of the source and the waves it causes is in agreement with the applicability of Lighthill's analogy, with no significant back-reaction of sound on the flow [1]. Excluding the source term at first, the simplest solution of Equation (8) has the form of a plane wave, the real part of

$$
\bar{h}^{\alpha \beta}=A^{\alpha \beta} e^{j k_{v} x^{v}} .
$$

Components of the polarization tensor $A^{\alpha \beta}$ are complex constants and the four-vector $k^{\alpha}$ is null vector in the flat Minkowski spacetime: $k_{\alpha} k^{\alpha}=\eta_{\alpha \beta} k^{\beta} k^{\alpha}=0$. For example, if we suppose that the plane wave propagates in the direction of the $x^{3}$-axis, $k^{\alpha}=\left[\omega / c_{0}, 0,0, \omega / c_{0}\right], k_{\alpha}=\eta_{\alpha \beta} k^{\beta}=$ $\left[-\omega / c_{0}, 0,0, \omega / c_{0}\right]$, where $\omega$ denotes angular frequency of the wave, and we obtain the usual exponent $j k_{v} x^{v}=-j \omega\left(t-\frac{x^{3}}{c_{0}}\right)$ after replacing $x^{0}$ with $c_{0} t$.

Plane transverse gravitational waves are typically analyzed in the transverse-traceless gauge which suppresses the longitudinal component and leaves only two non-zero transverse components of the polarization tensor. More suitable for longitudinal acoustic waves in fluids is the Newtonian gauge. The reason is that, unlike a relativistic observer, an acoustic receiver does not exist in the analogue 
acoustic spacetime, but in the Newtonian space and time. As a consequence, the Newtonian frame is preferred and only in this particular gauge the metric perturbation obtains classical acoustic meaning. In the theory of gravity such a gauge is used for calculations of the corrections of classical Newtonian gravitational potential due to relativistic phenomena. In order to show how it describes sound waves, we observe motion of a free particle in the acoustic spacetime. As in general relativity, it is given by the geodesic equation. When the particle is moving with velocity small compared to $c_{0}$ in an essentially flat spacetime, the three-dimensional acceleration equals to the lowest order [2]

$$
\frac{d^{2} x^{k}}{d t^{2}}=-\frac{c_{0}^{2}}{2} \eta^{k l}\left(h_{l 0,0}+h_{0 l, 0}-h_{00, l}\right)
$$

The condition for a slowly moving (non-relativistic) particle is satisfied by weak acoustic waves, since the particle velocity is much smaller than the speed of sound. In the Newtonian form $\left|h_{l 0}\right|=$ $\left|h_{0 l}\right| \ll\left|h_{00}\right|$ and therefore

$$
\frac{d^{2} x^{k}}{d t^{2}}=\frac{c_{0}^{2}}{2} h_{00}^{, k}
$$

Motion of the particle due to the wave thus depends on a single scalar $h_{00}$, as we expect from the longitudinal sound waves in fluids. In this way we can obtain a measurable acoustic quantity (acceleration) from the acoustic spacetime analogy. It follows that the Lorenz gauge is necessary as a prerequisite for the wave equation of $\bar{h}^{\alpha \beta}$, Equation (8), but once $\bar{h}^{\alpha \beta}$ has been obtained it has to be converted to $h^{\alpha \beta}$ and expressed in the Newtonian gauge in order to calculate the acoustic quantities such as particle velocity and sound pressure. Nevertheless, a purely geometric quantity, the metric perturbation, suffices for the description of sound waves and no dynamic analogy with linear general relativity is necessary.

\section{Aeroacoustic Sound Generation}

In the previous section we showed how a metric perturbation captures a sound wave in the acoustic spacetime. In this section we inspect the source of waves represented by the term on the right-hand side of Equation (8). We consider only non-relativistic flows in which all particles satisfy the condition $|\vec{v}| \ll c_{0}$ even in the source region, with $\vec{v}$ denoting three-dimensional particle velocity. Then, the approximation $\vec{U} \approx\left[1, \vec{v} / c_{0}\right]=\left[1, v^{1} / c_{0}, v^{2} / c_{0}, v^{3} / c_{0}\right]$ holds for four-velocity and the stress-energy tensor of a perfect fluid in nearly flat spacetime equals [2]

$$
T^{\alpha \beta}=\left[\begin{array}{cccc}
\rho_{0} c_{0}^{2} & \rho_{0} c_{0} v^{1} & \rho_{0} c_{0} v^{2} & \rho_{0} c_{0} v^{3} \\
\rho_{0} c_{0} v^{1} & \rho_{0} v^{1} v^{1}+p & \rho_{0} v^{1} v^{2} & \rho_{0} v^{1} v^{3} \\
\rho_{0} c_{0} v^{2} & \rho_{0} v^{1} v^{2} & \rho_{0} v^{2} v^{2}+p & \rho_{0} v^{2} v^{3} \\
\rho_{0} c_{0} v^{3} & \rho_{0} v^{1} v^{3} & \rho_{0} v^{2} v^{3} & \rho_{0} v^{3} v^{3}+p
\end{array}\right],
$$

where $\rho_{0}$ is matter density (here density of the background fluid) and $p$ is pressure. This stress-energy tensor satisfies the local conservation of mass and momentum:

$$
T_{, \beta}^{\alpha \beta}=0,
$$

which is a compact form of the laws of conservation of mass and momentum in an incompressible inviscid fluid. We notice the similarity of the spatial part $T^{j k}$ and isentropic Lighthill's tensor [1], which is the free-space aeroacoustic source of sound. The condition $|\vec{v}| \ll c_{0}$ with $c_{0}$ the speed of sound (which is the maximum speed in the theory) is satisfied in incompressible low Mach number flows, which are assumed by Lighthill's eighth power law.

However, a conceptually important difference compared with Lighthill's derivation is that we treat $T^{\alpha \beta}$ as the source of a purely geometric perturbation of the background acoustic spacetime, not as the source of acoustic pressure or density perturbation. We do not split the conservation equations, Equation (13), into the source and propagation parts, nor do we need to select a dynamic variable 
for describing acoustic waves. Lighthill's tensor follows from the conservation laws after the weak acoustic terms are shifted to the left-hand side of the wave equation to represent propagating sound waves. Therefore, its components are not fully conserved. In contrast to this, the entire stress-energy tensor which we consider here as the source satisfies the conservation laws in Equation (13). It is the source of perturbation of the spacetime itself, so it does not have to be split into the source and propagation parts in terms of dynamic quantities.

In the further analysis of wave generation we follow Misner et al. [12] and consider a single isolated source, far from which the acoustic spacetime is asymptotically flat towards the infinity. A small metric perturbation is defined in Equation (3) everywhere (including the source region) and Equation (8) holds under the condition in Equation (5). We also expect that only a small fraction of the stress-energy tensor is responsible for the radiation of waves, in accordance with the inefficiency of the mechanism of quadrupole radiation. Hence, it can be formally split into the dominant effective stress-energy tensor, $T_{\alpha \beta}^{\text {eff }}$, and the small component $t_{\alpha \beta}: T_{\alpha \beta}=T_{\alpha \beta}^{\text {eff }}+t_{\alpha \beta}$, and we can write

$$
\square \bar{h}_{\alpha \beta}=-\frac{2 k G}{c_{0}^{4}}\left(T_{\alpha \beta}^{\mathrm{eff}}+t_{\alpha \beta}\right) .
$$

The general solution for both ingoing $(\epsilon=-1)$ and outgoing $(\epsilon=+1)$ wave is

$$
\bar{h}_{\alpha \beta}=\frac{k G}{2 \pi c_{0}^{4}} \int \frac{\left[T_{\alpha \beta}^{\text {eff }}+t_{\alpha \beta}\right]_{\left(t-\epsilon R / c_{0}\right)}}{R} d^{3} \vec{y},
$$

where the integration is performed over the entire three-dimensional space and $R=\left|x^{i}-y^{i}\right|$ with $x^{i}$ location of the receiver. The values of $T_{\alpha \beta}^{\text {eff }}$ and $t_{\alpha \beta}$ are to be evaluated at the time $t-\epsilon R / c_{0}$.

Next we assume that the source is compact, so that its characteristic length scale satisfies $L \ll$ $c_{0} / \omega$, where $\omega$ is angular frequency of the oscillations. This actually comes down to the same slow-motion condition as above, $|\vec{v}| \ll c_{0}$, since $|\vec{v}| \sim L \omega$. We also consider geometric far field $(R \gg L)$, so we can approximate

$$
\bar{h}_{\alpha \beta}=\frac{k G}{2 \pi r c_{0}^{4}} \int\left[T_{\alpha \beta}^{\mathrm{eff}}+t_{\alpha \beta}\right]_{\left(t-\epsilon r / c_{0}\right)} d^{3} \vec{y},
$$

where $r$ is radial coordinate of the spherical coordinate system with the compact source in its origin.

From the conservation laws in Equation (13) we deduce [12]

$$
\frac{1}{c_{0}^{2}} \frac{d^{2}}{d t^{2}} \int\left(T_{00}^{\text {eff }}+t_{00}\right) x_{j} x_{k} d^{3} \vec{y}=2 \int\left(T_{j k}^{\text {eff }}+t_{j k}\right) d^{3} \vec{y} .
$$

This important identity relates spatial components of the stress-energy tensor, which closely correspond to Lighthill's tensor, with the component $T_{00}$ and accordingly removes the need for a split in Lighthill's derivation. It is possible exactly due to the fact that the full stress-energy tensor satisfies the conservation laws. The second-order time derivative on the left-hand side of Equation (17) takes over the role of the second-order derivatives of the source terms in Lighthill's analogy, which naturally appear when a second-order tensor reduces to a scalar, and ultimately determines the scaling law for the quadrupole source, one of the key results of Lighthill's original paper [1]. The integral on the left-hand side of Equation (17) represents the second moment of the mass distribution and multiplied by $1 / c_{0}^{2}$ it is called quadrupole moment tensor of the mass distribution. It is commonly denoted with $I_{j k}$, which is, thus, by definition

$$
I_{j k}=\frac{1}{c_{0}^{2}} \int\left(T_{00}^{\text {eff }}+t_{00}\right) x_{j} x_{k} d^{3} \vec{y} .
$$

The quantity which appears to be more convenient for mathematical description of wave generation is reduced quadrupole moment defined as 


$$
t_{j k}=I_{j k}-\frac{1}{3} \delta_{j k} I_{l}{ }^{l}
$$

where $\delta_{j k}$ is Kronecker delta. From Equations (16)-(18),

$$
\bar{h}_{j k}=\frac{k G}{4 \pi r c_{0}^{4}} \frac{d^{2}}{d t^{2}} I_{j k}\left(t-\epsilon r / c_{0}\right) .
$$

The assumption of geometric far field (with respect to the characteristic length scale of the flow in the source region) is necessary for simplifying the calculation with Equation (16). On the other hand, the assumption of acoustic far field, defined with respect to the sound wavelength, is not strictly necessary for estimating power of the quadrupole source. It is sufficient to calculate reaction of the source to the far-field radiation, which can be done in the acoustic near field. Expanding $\bar{h}_{j k}$ in powers of $r$ for $\omega r / c_{0} \ll 1$ (while $r \gg L$ ) and leaving only the terms with $\epsilon$, which correspond to the wave radiation, gives (after replacing $\epsilon=1$ for an outgoing wave)

$$
\bar{h}_{j k}^{\text {react }}=-\frac{k G}{4 \pi c_{0}^{5}} \frac{d^{3}}{d t^{3}} I_{j k}(t)-\frac{k G}{24 \pi c_{0}^{7}} r^{2} \frac{d^{5}}{d t^{5}} I_{j k}(t),
$$

where all higher-order terms of the series have been omitted. Using Equation (5), we can also find

$$
\bar{h}_{0 j}^{\text {react }}=-\frac{k G}{12 \pi c_{0}^{6}} x^{k} \frac{d^{4}}{d t^{4}} I_{j k}(t)-\frac{k G}{120 \pi c_{0}^{8}} r^{2} x^{k} \frac{d^{6}}{d t^{6}} I_{j k}(t)
$$

and

$$
\bar{h}_{00}^{\text {react }}=-\frac{k G}{12 \pi c_{0}^{5}} \frac{d^{3}}{d t^{3}} I_{j j}(t)-\frac{k G}{120 \pi c_{0}^{7}}\left(r^{2} \delta^{j k}+2 x^{j} x^{k}\right) \frac{d^{5}}{d t^{5}} I_{j k}(t)
$$

These components are radiation reaction potentials given in the Lorenz gauge. The omitted terms which do not correspond to the radiation (not involving $\epsilon$ ) represent incompressible fluctuations which do not propagate into the far field.

In order to calculate the Newtonian form which is suitable for obtaining classical acoustic quantities, we first switch back to $h_{\alpha \beta}=\bar{h}_{\alpha \beta}-\bar{h}^{v}{ }_{v} \eta_{\alpha \beta} / 2$ from Equation (7) and change the coordinates as $x^{\mu} \rightarrow x^{\mu}+\xi^{\mu}$, with

$$
\xi_{0}=-\frac{k G}{12 \pi c_{0}^{4}} \frac{d^{2}}{d t^{2}} I_{l l}(t)+\frac{k G}{48 \pi c_{0}^{6}} x^{j} x^{k} \frac{d^{4}}{d t^{4}} I_{j k}(t)-\frac{k G}{48 \pi c_{0}^{6}} r^{2} \frac{d^{4}}{d t^{4}} I_{l l}(t)
$$

and

$$
\xi_{j}=-\frac{k G}{8 \pi c_{0}^{5}} x^{k} \frac{d^{3}}{d t^{3}} I_{j k}(t)+\frac{k G}{24 \pi c_{0}^{5}} x^{j} \frac{d^{3}}{d t^{3}} I_{l l}(t)
$$

In this gauge [12],

$$
h_{00}^{\text {react }}=-\frac{k G}{20 \pi c_{0}^{7}} x^{j} x^{k} \frac{d^{5}}{d t^{5}} t_{j k}(t)
$$

at the leading order, while the components $h_{0 j}^{\text {react }} \sim\left(\omega L / c_{0}\right) h_{00}^{\text {react }}$ are of higher order and negligible for the supposed compact source. Therefore, we obtain the metric component $h_{00}$ in the Newtonian gauge, which describes the longitudinal perturbation of the acoustic spacetime due to the isentropic quadrupole source. Equation (26) is also one of the key results in the linearized theory of gravitation, which corrects the Newtonian gravitational potential with the contribution of gravitational wave radiation [12]. The geodesic Equation (11) gives the acceleration of a particle affected by the incoming wave:

$$
\frac{d^{2} x^{l}}{d t^{2}}=\frac{c_{0}^{2}}{2} h_{00}^{\text {react }, l}=-\frac{k G}{40 \pi c_{0}^{5}}\left(x^{j} x^{k} \frac{d^{5}}{d t^{5}} f_{j k}(t)\right)^{l} .
$$


We can now obtain a scaling law for the source power. Without considering details of the flow in the source region, we follow Lighthill and suppose that $T_{j k}$ scales as $\rho_{0}|\vec{v}|^{2}$, where $\rho_{0}$ is density of the essentially incompressible flow. From Equations (17)-(19), $\left|f_{j k}\right| \sim \rho_{0} L^{5}$, so the acoustic particle velocity from Equation (27) scales as

$$
\left|\vec{v}_{\mathrm{ac}}\right| \sim \frac{k G \rho_{0} L^{2}}{c_{0}^{5}}(\omega L)^{4}
$$

Sound intensity scales as

$$
|\vec{I}| \sim \rho_{0} c_{0}\left|\vec{v}_{\mathrm{ac}}\right|^{2} \sim \frac{k^{2} G^{2} \rho_{0}^{3} L^{4}}{c_{0}}\left(\frac{|\vec{v}|}{c_{0}}\right)^{8}
$$

where we also replac $\omega L \sim|\vec{v}|$. Thus, we obtain the eighth power law for the acoustic power of the quadrupole source, in agreement with the result of Lighthill [1].

A more detailed comparison leads to one more interesting result. After replacing $r \sim L$ in the considered acoustic near field, Lighthill's power law gives the scaling $[1,5]|\vec{I}| \sim \rho_{0} c_{0}^{3}\left(|\vec{v}| / c_{0}\right)^{8}$. If we further ignore the multiplication constant which is determined by the dimensionless $k$, this matches Equation (29) if we set

$$
G=\frac{c_{0}^{2} L}{2 M} \sim \frac{c_{0}^{2}}{2 \rho_{0} L^{2}}
$$

where $M$ is total mass of the source. In this way we can identify length scale of the source $L$ as the acoustic Schwarzschild radius,

$$
L=\frac{2 G M}{c_{0}^{2}} .
$$

In cosmology, Schwarzschild radius determines length scale of a source of gravitational waves, such as a rotating black hole in a black-hole binary. The same concept appears to characterize length scale of the source of waves in the analogue acoustic spacetime.

It is worth mentioning that compact sources of longitudinal waves are much less efficient than sources of transverse waves. The reason is that the second-order time derivative in Equations (17) and (20) leaves the multiplication factor $\left(\omega L / c_{0}\right)^{2}$ in the expression for wave amplitude and $\left(\omega L / c_{0}\right)^{4}$ for radiated power, with $\omega L / c_{0} \ll 1$. In fact, the weak longitudinal component is completely removed in the transverse-traceless gauge, which is typically used for transverse gravitational waves in the linearized theory. However, the fluids do not support propagation of transverse acoustic waves and the remaining longitudinal waves obey the eighth power law.

\section{Conclusions}

Although Lighthill's power law for a quadrupole source in an inviscid incompressible flow is merely reproduced using the formalism of the acoustic spacetime analogy, the derivation given above has several important implications. First, the description of sound generation in acoustic spacetime appears to be more natural than the classical derivation based on the conservation laws. The entire stress-energy tensor is taken as the source of waves, without splitting it into the source and propagation terms or selecting an appropriate dynamic quantity (acoustic pressure or density) for the aeroacoustic analogy. Second, generated sound waves are treated as a purely geometric perturbation of the background acoustic spacetime, described by the weak unsteady component of the metric tensor. Rather than contracting the second-order tensor in the source term with double divergence, the entire metric tensor field associated with sound waves is observed and the acoustic scalar component is extracted by the choice of Newtonian gauge. Third, the derivation of the eighth power law proves that the acoustic spacetime analogy can be extended beyond sound propagation to aeroacoustic sound generation, at least in incompressible flows where the kinematic effects dominate. Hence, the acoustic analogy covers not only the background metric, but the sound waves and kinematic sources, as well. 
The applied methodology can include sound propagation effects in a non-uniform flow outside the source region, such as convection and refraction. The Minkowski metric $\eta^{\alpha \beta}$ in Equation (3) should be replaced accordingly with an appropriate background metric [10]. These effects cannot be retrieved from the analogy with gravitation, since curvature of the background acoustic spacetime depends on the state of the external steady flow, not mass itself. The acoustic analogy indeed captures kinematics and not dynamics of general relativity. However, this does not imply that the Einstein field equations with the source term cannot be used for capturing unsteady changes of the acoustic spacetime, as demonstrated above. This is possible exactly because sound generation in an incompressible flow is purely kinematic and the stress-energy tensor reduces to the same form in both theories for non-relativistic velocities.

The analysis was based on the linearized theory of weak perturbation and free-space sound generation in low Mach number flows. Further investigation is necessary in order to check whether the analogy can be extended to include certain non-linear acoustic phenomena and high Mach number flows, or the effects of boundaries in the flow, such as reduction of aeroacoustic quadrupole to dipole, although in such case the analogy with covariant electromagnetism appears to be more natural. The obtained results also open the possibilities for further studies of the analogy between vortex pairs, as compact aeroacoustic sources, and rotating black-hole binaries, as typical sources of gravitational waves.

Author Contributions: Conceptualization, D.M.; methodology, D.M.; validation, E.S.; writing—original draft preparation, D.M.; supervision, E.S. All authors have read and agreed to the published version of the manuscript.

Funding: This research received no external funding.

Conflicts of Interest: The authors declare no conflict of interest.

\section{References}

1. Lighthill, M.J. On sound generated aerodynamically, I. General theory. Proc. R. Soc. Lond. 1952, A211, 564-586.

2. Schutz, B. A First Course in General Relativity, 2nd ed.; Sections 8 and 9; Cambridge University Press: Cambridge, UK, 2017.

3. Gordon, W. Zur Lichtfortpflanzung nach der Relativitätstheorie ("Towards propagation of light based on the theory of relativity"). Ann. Phys. 1923, 72, 421-456. [CrossRef]

4. Jones, D.S. Acoustic and Electromagnetic Waves; Oxford University Press: Oxford, UK, 1986; pp. 10-14.

5. Rienstra, S.W.; Hirschberg, A. An Introduction to Acoustics; Sections 6.5 and 9.1; Eindhoven University of Technology: Eindhoven, The Netherlands, 2018.

6. Unruh, W.G. Experimental black-hole evaporation. Phys. Rev. Lett. 1981, 46, 1351-1353. [CrossRef]

7. Barceló, C.; Liberati, S.; Visser, M. Analogue gravity. Living Rev. Relativ. 2011, 14, 1-151. [CrossRef] [PubMed]

8. Barceló, C.; Liberati, S.; Sonego, S.; Visser, M. Causal structure of analogue spacetimes. New J. Phys. 2004, 6, 1-48. [CrossRef]

9. Pierce, A.D. Wave equation for sound in fluids with unsteady inhomogeneous flow. J. Acoust. Soc. Am. 1990, 87, 2292-2299. [CrossRef]

10. Gregory, A.; Agarwal, A.; Lasenby, J.; Sinayoko, S. Geometric algebra and an acoustic space time for propagation in non-uniform flow. In Proceedings of the 22nd International Congress on Sound and Vibration (ICSV), Florence, Italy, 12-16 July 2015.

11. Bergliaffa, S.E.P.; Hibberd, K.; Stone, M.; Visser, M. Wave equation for sound in fluids with vorticity. Physica D 2004, 191, 121-136. [CrossRef]

12. Misner, C.W.; Thorne, K.S.; Wheeler, J.A. Gravitation; Sections 35 and 36; Princeton University Press: Princeton, NJ, USA, 2017.

(C) 2020 by the authors. Licensee MDPI, Basel, Switzerland. This article is an open access article distributed under the terms and conditions of the Creative Commons Attribution (CC BY) license (http://creativecommons.org/licenses/by/4.0/). 